\title{
6 Wie Literatur angewandt wird
}

Die Arbeit sollte zeigen, dass es Applikationen in dem erläuterten Sinne beim außerwissenschaftlichen Umgang mit Literatur in Geschichte und Gegenwart gibt, wie solche Applikationen beschaffen sind und dass es sich um eine relevante Praktik handelt. Bei allen neun Romanen lassen sich in unterschiedlich großer Zahl Applikationen identifizieren. Den Gang der Untersuchung rekapitulierend, sollen zunächst die wesentlichen Ergebnisse zusammengefasst werden, bevor dann einige darauf aufbauende systematische Erträge angedeutet und weiterführende Gesichtspunkte erwähnt werden können.

\subsection{Zusammenfassung der Ergebnisse}

Die Leiden des jungen Werthers gaben in der Wahrnehmung der Zeitgenossen, soweit sie sich in Rezeptionsdokumenten äußerten, die überliefert sind, Anlass zu mehreren Applikationen. Eine erste hat mit dem Selbstmord des Protagonisten und der darauf möglichen Perspektive zu tun. Die Romanfigur erlaubte mehr oder weniger komplexe Einsichten in die Psyche realer Menschen. Der Roman konnte geeignet sein, wenn nicht die Taten, so doch die Menschen einer milderen moralischen Beurteilung zugänglich zu machen und eine von Mitleid geprägte Einstellung zu ihnen zu befördern. Gerade befürchtete ethische Applikationen riefen Zeitgenossen auf den Plan, die in zumeist literarischen Gegenschriften eine alternative Sicht formulierten, bei welcher der Selbstmord als falsch und nicht ratsam erscheinen sollte. Die Absicht bestand darin, eine alternative Applikation anzubieten und eine negative Applikation des in Goethes Roman Dargestellten zu bewirken. In ähnlicher Weise wurde eine zweite Applikation befürchtet, nämlich, dass Werthers Lebenswandel und seine Überzeugungen als Vorbild dienen konnten - eine Annahme, die in der Sache die Vorstellung einschließt, dass das Gelesene in dieser Hinsicht appliziert wird. Tatsächlich gab es Stimmen, die Werther zu einem Ideal erklärten.

Wo Werther gleichsam anthropologische oder philosophische Aussagen über den Menschen und menschliches Leben in den Mund gelegt werden, gab es, drittens, bei manchen Zeitgenossen die Tendenz, sie als zutreffend und profund anzusehen, während andere an derartigen Aussagen und zumal an Werthers religiösen Ansichten Anstoß nahmen und Gegenschriften publizierten. Wo also die einen die Figurenrede applizierten, schrieben die anderen gegen derartige Applikationen und vermeintliche weitere an. Eine vierte Applikation hat damit zu tun, wie der Protagonist mit seinen Gefühlen umgeht und wie 
sie sein Handeln bis hin zum Selbstmord bestimmen. Die einen gelangten zu der Überzeugung, der Roman zeige in zutreffender Weise, dass es Fälle geben kann, in welchen die Gefühle stärker sind als die rationalen Vermögen des Menschen. Der Roman ermögliche, so meinten einige, Einsichten in die Natur, die Entwicklung und die Auswirkungen von Gefühlen. Andere bestritten genau dies, hielten also eine in ihrer Wahrnehmung vom Roman nahegelegte Sicht für unzutreffend. Fünftens schließlich konnte Werther so gelesen werden, dass er ein Liebesideal darstelle oder vor den negativen Folgen einer unglücklichen Liebe warne. Einer solchen Applikation standen Negativapplikationen gegenüber, bei denen der Nachweis zu führen versucht wurde, dass die im Roman dargestellte Liebe gerade nicht als Ideal aufzufassen sei.

Millers Siegwart weist, wie gezeigt werden konnte, ein relativ eindeutiges, einheitliches und den gesamten Text bestimmendes Applikationspotenzial auf. Der Roman zeigt eine mögliche Konzeption von Liebe und präsentiert sie als erstrebenswertes Ideal, das sich die Rezipient/-innen zu eigen machen konnten. $\mathrm{Zu}$ dieser Schlussfolgerung berechtigen zahlreiche Textbefunde, aus denen hervorgeht, dass und wie in dem Roman Liebe thematisiert wird. In durchaus origineller Weise erfüllt der Text die zeitgenössisch und von Miller postulierte Funktion, zu unterrichten und zu belehren.

Ist bei diesem Roman der Text selbst, in Verbindung mit den historischen Gegebenheiten, sehr aufschlussreich in Bezug auf die Applikation, so sind es im Falle von Lafontaines Klara du Plessis und Klairant Selbstaussagen des Autors, die als Indizien für Applikationen angesehen werden können. Lafontaine schrieb Romanen unter anderem die Funktion zu, eine angemessene Auffassung von Liebe zu vermitteln, und zu zeigen, wie man mit ihr umzugehen habe. Eine Untersuchung der Beschaffenheit des Textes führt, ähnlich wie bei Miller, zu dem Ergebnis, dass die Darstellung und Perspektivierung einer Liebesgeschichte durchaus geeignet ist, solche Absichten und damit die intendierte Applikation zu realisieren. Der Roman konnte Einsichten vermitteln, was Liebe sei und wie ein angemessener Umgang mit ihr aussieht.

An den drei Romanen aus dem 18. Jahrhundert lassen sich exemplarisch drei systematische Perspektiven auf das Phänomen der Applikation verdeutlichen. Die wohl einmalig gut zu nennende Quellenlage bei der Erstrezeption von Goethes Werther macht diesen Text in der Tat $\mathrm{zu}$ einem rezeptionsgeschichtlichen Musterfall, insbesondere mit Blick auf Applikationen. Von den Befunden lässt sich nicht ohne Weiteres auf die Rezeption anderer zeitgenössischer Romane schließen. Art und Umfang der festgestellten Applikationen sind jedoch ein gewichtiges Argument für die Existenz und Relevanz von Applikationen beim außerwissenschaftlichen Umgang mit Literatur. Außerdem verdeutlicht 
das Beispiel, wie aussagekräftig eine detaillierte und umfassende Untersuchung der tatsächlichen Rezeption, hier mit Blick auf die Applikation, sein kann. An Millers Siegwart wird ersichtlich, dass und wie es aufschlussreich sein kann, einen literarischen Text in seinen historischen Zusammenhängen hinsichtlich seines Applikationspotenzials, also eines Wirkungspotenzials, zu interpretieren. Es zeigt sich in dem konkreten Fall, dass der Roman in darstellungstechnischer, rhetorischer wie pragmatischer Hinsicht plausiblerweise die identifizierte Applikation hervorrufen konnte. Ob und in welchem Umfang dieses Potenzial im Einzelnen realisiert wurde, lässt sich aufgrund der Quellenlage nicht genau sagen. Dass der Roman so appliziert werden konnte, geht aus einzelnen Quellen jedoch hervor. Wie am Beispiel von Lafontaines Klara du Plessis und Klairant gezeigt, können Selbstaussagen der Autor/-innen ebenfalls bedeutsam sein. Betrachtet man den Text vor dem Hintergrund dessen, was man als intendierte Applikation des Autors rekonstruieren kann, dann zeigt sich, dass er ein hohes $\mathrm{Maß}$ an Eignung für eine solche Applikation aufweist. Absichten der Autor/innen, Potenziale der Texte und - hier besonders wichtig - die tatsächliche Rezeption sind prima facie gleichermaßen bedeutsame Faktoren, die bei einer literaturwissenschaftlichen Untersuchung des Umgangs mit Literatur, wie er in Geschichte und Gegenwart praktiziert wird, nicht gegeneinander ausgespielt werden sollten, da sie potenziell einen Aussagewert besitzen und gerade der Vergleich relevante Einsichten ermöglicht.

Reuters Aus guter Familie hat zeitgenössisch mindestens drei (Gruppen von) Applikationen hervorgerufen. Die große Mehrheit stellte das Gelesene in einen Zusammenhang mit der sogenannten ,Frauen-“ oder ,Mädchenfrage‘. Der Roman wurde als zutreffende Darstellung einer relevanten Problematik angesehen, nämlich der Frage, wie in Familie und Gesellschaft mit unverheirateten bürgerlichen Frauen umgegangen wird. Er konnte als Kritik an der Erziehung bürgerlicher Mädchen und an den bürgerlichen Verhaltensnormen aufgefasst werden. Darüber hinaus wurde die Schilderung der Protagonistin als korrekte Darstellung der psychischen Befindlichkeiten mancher Frauen angesehen. Schließlich wurde das Gelesene so wahrgenommen, dass es zu handlungspraktischen Konsequenzen bei der Erziehung und allgemein beim Umgang mit unverheirateten bürgerlichen Frauen Anlass gebe. Eine demgegenüber deutlich kleinere Gruppe applizierte den Roman in einem weiterreichenden emanzipatorischen Sinne. In ihrer Wahrnehmung gab der Roman Anlass, Überzeugungen und Einstellungen grundlegender zu ändern. Der Text wurde im Zusammenhang gesehen mit Forderungen nach Gleichberechtigung, freier Berufswahl und selbstbestimmter Sexualität. Eine ebenfalls kleinere Gruppe lehnte Applikationen ab, die auf die geschlechtergeschichtlichen Verhältnisse bezogen waren, 
applizierte den Roman aber ebenfalls, nämlich als Darstellung der Unzulänglichkeit der Welt oder als Einsicht in das vermeintliche Wesen des Weiblichen und seine Tragik. Die Autorin selbst wollte ihren Roman als Darstellung einer von sozialen und historischen Bezügen losgelösten ,rein menschlichen“ Tragik verstanden wissen: Der Roman zeige das Leiden unverheirateter bürgerlicher Frauen, insbesondere solcher mit einer bestimmten charakterlichen Disposition. Das Gelesene als realweltlich zutreffend anzusehen und als ,tragisch“ aufzufassen, bedeutet, den Text in diesen beiden Hinsichten zu applizieren. Erklären lassen sich alle diese Applikationen durch Verweis auf die Beschaffenheit des Textes. Die Applikationen der ersten Gruppe kommen dabei am ehesten als Realisierungen des Applikationspotenzials des Textes in Betracht, die anderen beiden mögen in einem schwächeren Sinne von der Beschaffenheit des Textes verursacht worden sein und dürften sich in erster Linie lebensweltlichen Einstellungen verdanken, welche die Lektüre beeinflussen.

Manns Buddenbrooks wurden zeitgenössisch so appliziert, dass der Text eine pessimistische Sicht auf ,das Leben“ vermittele. Der Roman zeige das Leben in realweltlich zutreffender Weise als blinde und unverständliche Macht, welcher der einzelne Mensch schutzlos ausgeliefert ist. Mann selbst hat eine solche Applikation vorgenommen, die sich durchaus als Realisierung eines Applikationspotenzials des Textes auffassen lässt. Außerdem hat er im Verlauf der Jahrzehnte verschiedene, deutlich weniger gut auf den Text zurückzuführende Applikationen vorgeschlagen. Der Roman verdeutliche eine Entwicklung im Bürgertum hin zu einer ,Entbürgerlichung،, er stelle Thomas als ,Leistungsethiker‘ dar, wie ihn die zeitgenössische Soziologie entdeckt hatte, er zeige einen spezifisch deutschen Prozess der décadence. Ein naheliegendes Applikationspotenzial des Textes scheint zeitgenössisch, soweit sich das sagen lässt, nicht realisiert worden zu sein. Aufgrund seiner Beschaffenheit gibt der Roman Anlass, Relevanz und Problematik von milieu- und geschlechtsspezifischen Rollenerwartungen zu erkennen.

Mit Im Westen nichts Neues verfolgte Remarque nach eigener Aussage im Wesentlichen zwei Absichten, deren erfolgreiche Realisierung eine Applikation zur Voraussetzung hat. Er wollte auf die Probleme aufmerksam machen, welche die Generation, die in relativ jungen Jahren am Krieg teilnahm, bei der Wiedereingliederung in die Nachkriegsgesellschaft hatte. Für viele von ihnen, so Remarques Überzeugung, bedeutete der Krieg eine Zäsur, welche das weitere Leben nachhaltig beeinträchtigte. Dies zu zeigen und um Verständnis für die Betroffenen zu werben, war eine erste intendierte Applikation. Eine zweite Applikation bezog sich auf die Betroffenen selbst. Ihnen sollte verdeutlicht werden, dass der Kriegseinsatz die Ursache ihrer Probleme war, und lebensprakti- 
sche Hilfe angeboten werden. Nach Aussage des Autors wurden beide Applikationen tatsächlich realisiert. Der Text verfügt in dieser Hinsicht durchaus über ein Applikationspotenzial, allerdings nur bedingt: Das Entscheidende, nämlich die Schwierigkeiten in der Nachkriegsgesellschaft, werden nur allgemein benannt, nicht jedoch gezeigt. Nun mag das für die Zeitgenossen, zumal die Betroffenen, nicht erforderlich gewesen sein, um den Roman zu applizieren, da sie es aus eigener Anschauung oder Erfahrung kannten. Möglicherweise liegt hierin allerdings ein Grund, warum die überwiegende Zahl der in den Rezeptionsdokumenten nachweisbaren Applikationen in eine andere Richtung geht.

Als entscheidend erwies sich zum einen die Frage, ob der Roman den Stellungskrieg an der Westfront zutreffend und authentisch schildere. Sie wurde kontrovers diskutiert und ebenso vehement bejaht wie verneint. Damit wurde das im Roman Dargestellte positiv oder negativ appliziert. Wo man es positiv applizierte, wurde bisweilen hervorgehoben, dass der Roman der Erinnerung dienen könne, identifikatorische Lesarten ermögliche und zeige, wie menschliches Erleben in der Extremsituation des Krieges beschaffen sei. Zum anderen gab es unterschiedliche Auffassungen in der Frage, welche Konsequenzen daraus $\mathrm{zu}$ ziehen seien, dass der Roman eine bestimmte Perspektive auf das Kriegsgeschehen vermittle. Der Roman konnte so verstanden werden, dass er eine pazifistische Haltung befördere oder, seltener, eine bellizistische.

Alle drei Romane aus der Epoche der literarischen Moderne geben in systematischer Hinsicht zugleich einen Eindruck davon, dass es bei der Rezeption und zumal der Applikation von Literatur von herausragender Bedeutsamkeit sein kann, den Text auf die alltägliche Erfahrungswirklichkeit zu beziehen. Für alle drei Romane ist es wesentlich, sie so zu lesen, dass sie etwas thematisieren, das zeitgenössisch in spezifischer Weise und besonderem Maße relevant war, und darauf eine Perspektive anbieten, die zu einer Stellungnahme herausforderte. In allen drei Fällen handelt es sich um solche Dimensionen historischer Wirklichkeit, die im Rahmen einer Historischen Anthropologie besonders zur Geltung kommen. Bei Reuter sind es die geschlechts- und milieuspezifischen Erfahrungen einer weiblichen Figur, welche aus der Innensicht geschildert werden, und somit auf der Mikroebene der Akteure, nicht der gesellschaftlichen Strukturen. Im Falle von Mann verweist die Erstrezeption wohl eher auf mentalitätsgeschichtlich beschreibbare Dispositionen als auf geistesgeschichtliche Positionen, welche den Charakter von Symptomen dieser Mentalität haben. Bei Remarque steht der soldatische Alltag und die Art und Weise, wie er an und hinter der Front erlebt werden konnte, im Vordergrund, nicht hingegen der Versuch einer ideologischen Sinngebung, eine Perspektive der militärischen Führung oder dergleichen. 
Süskinds Das Parfum wird in Rezensionen, die bei LovelyBooks eingestellt wurden, vor allem in drei Hinsichten zu applizieren versucht. Der Roman wird, erstens, so gelesen, dass er Einsichten in die Abgründe der menschlichen Psyche ermöglicht, die von der Sozialisation mitbedingt werden. Zweitens wird er so aufgefasst, dass er ein kulturgeschichtlich zutreffendes Bild alltäglichen Lebens im 18. Jahrhundert bietet. Drittens wird angenommen, dass das im Roman über Gerüche und Geruchssinn Gesagte in Teilen zutreffend ist und die eigene Wahrnehmung verändern kann. Mit Blick auf die Beschaffenheit des Textes erweisen sich solche Applikationen allerdings als eher partiell, unspezifisch und vom Text nur bedingt gedeckt. Sieht man einmal von dem eher einfachen Umstand ab, dass der Roman geeignet zu sein scheint, die olfaktorische Wahrnehmung zu beeinflussen, führt von dem, was im Roman über die Rolle und Funktion des Geruchssinns gesagt wird, kein direkter Weg zu lebensweltlichen Einsichten. Dafür ist das Geschilderte zu wunderbar. Was der Roman an korrekten kulturgeschichtlichen Details beinhaltet, dient der Konturierung des Schauplatzes und bleibt im Hintergrund. Entsprechende Applikationen sind partiell. Da der Protagonist nicht als realistische Figur konzipiert ist, eignet er sich nur bedingt für Einsichten in die menschliche Psyche. Da er gleichsam ,böse geboren‘ ist, fungiert er nicht als Beispiel für das Monster, das eigentlich die monströsen gesellschaftlichen Zustände anschaulich macht. Bestenfalls liegen daher Applikationen anhand des Textes vor. Überhaupt verfügt der Roman über ein geringes Applikationspotenzial. Dass sich dennoch Versuche nachweisen lassen, das Gelesene zu applizieren, kann als Indiz für die Relevanz dieser Lektürepraktik angesehen werden.

Schlinks Der Vorleser dagegen verfügt, aufgrund der Thematik und ihrer Darbietung wenig überraschend, über ein hohes Applikationspotenzial, welches in zahlreichen Rezensionen bei LovelyBooks realisiert zu werden scheint. Dazu gehört die Frage, wer die Täter waren, was sie bewog und wie sie zu beurteilen sind, der Generationenkonflikt und die Schuldproblematik, außerdem die Beziehung zweier ungleicher Partner und der Analphabetismus. Als zentral erweist sich das Dilemma, einen Menschen zu lieben, den man aufgrund seiner Beteiligung an den Verbrechen des NS eigentlich verurteilen will. Im Einzelfall wird das Dilemma erkannt, welches am ehesten mit dem Applikationspotenzial des Textes in Einklang zu bringen ist. Der Roman lässt sich so verstehen, dass er vor allem von diesem, letztlich emotionalen Dilemma handelt. Die Applikation besteht also darin, das Dilemma einer Generation zu erkennen, als lebensweltlich zutreffend anzusehen und anzuerkennen, also Verständnis für die betroffenen Angehörigen der zweiten Generation zu entwickeln. Die anderen auf den NS bezogenen Gesichtspunkte sind diesem Applikationspotenzial nachgeord- 
net. Applikationen, welche die Paarbeziehung und den Analphabetismus fokussieren, blenden deren Einbettung in die NS-Thematik aus, von welcher sie funktional abhängig sind. Es wird nicht beachtet, dass sie sich in diesem funktionalen Bezug erschöpfen.

Kehlmanns Die Vermessung der Welt weist ebenfalls ein hohes Applikationspotenzial auf. Die LovelyBooks-Rezensionen heben Einsichten in das Funktionieren von Wissenschaft und den Erwerb von Erkenntnis hervor oder betonen, dass der Roman Aufschluss gebe über den Typus des genialen Wissenschaftlers. Daneben kann er so gelesen werden, dass er Faktenwissen über die Zeit und die historischen Personen vermittelt, eine kritische Sicht auf Wissenschaft und die Zeit nahelege oder eine Satire auf das Deutschsein sei. So sehr sich solche Applikationen auf die Beschaffenheit des Textes zurückführen lassen, bleibt doch festzustellen, dass damit, ähnlich wie bei Schlinks Der Vorleser, nur Teile des Textes appliziert und das eigentliche Applikationspotenzial des Textes eher umkreist wird. Darin unterscheidet sich die Rezeption des Romans bei LovelyBooks nicht von der Rezeption im Feuilleton. Es lässt sich zeigen, dass der Roman vor allem eine distanzierte und ironische Perspektive auf die deutsche Geschichte entwirft. Sie erweist sich als notwendig, wenn man zum einen den NS nicht ausblenden oder relativieren, zum anderen aber die deutsche Geschichte davor nicht allein als Vorgeschichte des NS ansehen und ablehnen will. Sie ist dadurch Garant für eine ausgewogene Sicht auf die deutsche Geschichte und deren angemessene Beurteilung. Wenn man sich diese Perspektive zu eigen macht oder wenn man sie als unangemessen ablehnt, hat man den Roman appliziert.

In systematischer Hinsicht ist bei allen drei Romanen insbesondere das Verhältnis von Applikation und Applikationspotenzial aufschlussreich. Das Parfum verfügt über ein eher schwaches Applikationspotenzial, wird dafür jedoch verhältnismäßig oft zu applizieren versucht. Der Vorleser weist ein relativ großes Applikationspotenzial auf, welches realisiert wird. Für die zentrale, den Gesamttext berücksichtigende Applikationsmöglichkeit gilt das allerdings nur bedingt. Die Vermessung der Welt bietet sich ebenfalls für Applikationen an, die dafür aber eher selten nachgewiesen werden können. Das zentrale Applikationspotenzial scheint hier nicht erkannt worden zu sein. Der Befund, dass Applikationen bei LovelyBooks-Rezensionen vorkommen, berechtigt $\mathrm{zu}$ der Annahme, dass es sich nicht allein um ein Phänomen des professionellen außerwissenschaftlichen Umgangs mit Literatur handelt, sondern auch - und vielleicht gerade - des nicht-professionellen. Bei allen Unterschieden zwischen Applikationen, auf welche Rezensionen in Zeitungen und Zeitschriften hindeu- 
ten, und denen bei LovelyBooks, sind sie doch in manchen Hinsichten vergleichbar oder ähnlich.

\subsection{Weitergehende systematische Erträge}

Unabhängig davon, ob man die systematischen Befunde dieser Arbeit akzeptiert, lassen sich zunächst einige materielle Erträge festhalten, welche die zeitgenössische Rezeption der untersuchten Romane betreffen und das literaturwissenschaftliche Verständnis dieser Texte.

Die zeitgenössische Rezeption aller neun zum Korpus gehörenden Romane wurde von der bisherigen Forschung in unterschiedlichem Umfang untersucht. In der einen oder anderen Hinsicht können die Ergebnisse der vorliegenden Arbeit den Forschungsstand ergänzen oder korrigieren, wie zumindest angedeutet werden soll. Werther wurde zum Beispiel rezipiert hinsichtlich des Verhältnisses von ,Verstand“ und ,Leidenschaften', Siegwart mit Blick auf das Liebesideal, Klara du Plessis und Klairant erschöpfte sich nicht in dem zeitgeschichtlichen Bezug auf die Französische Revolution. Bei Reuters Aus guter Familie spielte emanzipatorische Kritik eine untergeordnetere Rolle, als man heute erwarten würde, oder sie fungierte als Feindbild. Der Roman wurde vor allem als Anlass zu moderater Reform betrachtet. Manns Buddenbrooks wurden vor dem Hintergrund der zeitgenössischen Mentalität rezipiert. Remarques Im Westen nichts Neues war deutlich umstrittener, als man aus heutiger Sicht vermuten würde. In Das Parfum wurde da, wo man sich nicht auf stilistische oder ästhetische Aspekte konzentrierte, durchaus nach über Unterhaltung hinausgehenden Themen gesucht. Der Vorleser wurde, anders als in Teilen der professionellen Literaturkritik befürchtet, wohl in der Mehrheit nicht zum Anlass genommen für revisionistische Sichtweisen auf die Geschichte des NS und bewirkte keine Exkulpierung der Täter. Die Vermessung der Welt wurde verschiedentlich wahrgenommen, ohne dass sich eine klare Tendenz ergab hinsichtlich der Frage, was das übergeordnete Thema des Textes ist.

Die tatsächliche Rezeption eines literarischen Textes ist analytisch von der Beschaffenheit und dem Potenzial des Textes zu unterscheiden. Dennoch kann die Rezeption bedeutsam sein für ein verbessertes literaturwissenschaftliches Verständnis der Texte. Das in Rezeptionsdokumenten zum Ausdruck kommende Verständnis des Gelesenen kann der Generierung von Interpretationshypothesen dienen, die sich literaturwissenschaftlich überprüfen lassen. Zum anderen gibt es einen sachlichen Zusammenhang. Nicht für alle Interpretationsziele, aber zumindest für manche der historischen, können die zeitgenössischen Rezipient/-innen in gewissem Maße als potenziell epistemisch privilegiert gegen- 
über der heutigen Wissenschaft angesehen werden. Als historische Akteure verfügen sie mit größerer Wahrscheinlichkeit über die relevanten Verstehensvoraussetzungen, welche die Literaturwissenschaft erst rekonstruieren muss. Vom heutigen Standpunkt aus neigt man aufgrund gewandelter Gegebenheiten eventuell dazu, die Texte in einer Weise zu lesen, die den historischen Verhältnissen nicht Rechnung trägt. Die Art und Weise, wie die Zeitgenossen einen literarischen Text aufnahmen, kann daher als Argument fungieren, um eine gegebene Interpretation zu stützen und vor Fehlinterpretationen zu schützen.

Die Untersuchung der Erstrezeption der neun Romane hat ferner zu einem besseren literaturwissenschaftlichen Verständnis der Texte beigetragen. Goethes Roman zeigt vor allem, wie der Protagonist mit seinen Gefühlen und insbesondere seiner Liebe zu Lotte umgeht. Millers Roman thematisiert in der Tat auf vielfältige Weise eine Liebeskonzeption. Lafontaines Roman behandelt tatsächlich den angemessenen Umgang mit Gefühlen der Liebe. Aus guter Familie gibt nicht definitiv zu erkennen, wie das Geschehen motiviert ist, und fordert dazu heraus, eine mögliche Motivierung zu finden. Buddenbrooks handelt von Rollenerwartungen und zeigt, wie die Figuren daran scheitern, diesen gerecht zu werden. Im Westen nichts Neues benennt explizit, was das Entscheidende ist, zeigt es aber nicht. Süskinds Das Parfum ist vor allem ein Unterhaltungsroman, der keine Botschaft enthält. Schlinks Der Vorleser veranschaulicht vor allem ein emotionales Dilemma, nicht ein ethisches. Kehlmanns Die Vermessung der Welt bietet eine ironisch-distanzierte Sicht auf die deutsche Geschichte an.

In Anbetracht der Befunde lässt sich sagen, dass es Applikationen in dem erläuterten Sinne beim außerwissenschaftlichen Umgang mit Literatur in Geschichte und Gegenwart gibt. Applikation ist eine relevante Praktik, die zu einem vollständigen Bild davon, was Literatur ist und wie mit ihr umgegangen wird, dazugehört. Sie ist, um es noch einmal zu betonen, nicht die einzige und nicht die dominierende. Es gibt aber keinen sachlichen Grund, warum sie in Konkurrenz stehen sollte zu anderen Rezeptionsweisen. Der Begriff der Applikation ist geeignet, einen Teil der empirisch vorkommenden Rezeption, wie er sich in Quellen beobachten lässt, zu klassifizieren und zu erklären. Davon dürften die neun Fallstudien, die als dichte Beschreibungen einer Praktik auf der Mikroebene literarischer Kommunikation angelegt waren, einen hinreichend deutlichen Eindruck geben. Die Ziele dieser Arbeit sind somit erreicht: Es konnte gezeigt werden, dass es Applikationen gibt, wie sie in konkreten Fällen beschaffen sind und dass sie relevant sind. Auf Grundlage der Ergebnisse sollen nun darüber hinaus weitergehende systematische Erträge umrissen werden, die über die Untersuchung der neun Romane hinausweisen. 
Verschiedentlich und so, dass sich ein Muster erkennen lässt, wird bei der Rezeption der Romane die Thematik und ihr lebensweltlicher Bezug positiv hervorgehoben oder das Fehlen eines relevanten, lebensnahen Themas beklagt. Es scheint ein ausgeprägtes Bedürfnis zu geben, Textwelt und Lebenswelt in Beziehung zueinander zu setzen. Das gilt im Prinzip für alle neun Romane und kann besonders deutlich werden an der Rezeption von Aus guter Familie und Im Westen nichts Neues. Ob das Geschilderte tatsächlich passieren kann, die Figuren wirklichen Menschen nahekommen, kurz: ob das Dargestellte als wahrscheinlich, natürlich, realistisch, glaubhaft angesehen werden kann, oder wie die Bezeichnungen lauten mögen, und was daraus für das Verständnis lebensweltlicher Sachverhalte folgt, ist häufig von Belang. Eigens hervorzuheben ist die Thematik: Es sind zwischenmenschliche Beziehungen, Innenansichten anderer Menschen, außergewöhnliche Extremsituationen und Probleme des Alltags, Mentalitäten und Identitäten, die besonders interessieren. Literarische Texte, so zeigt sich, werden bevorzugt deswegen gelesen, weil sie von Themen handeln, die von den Rezipient/-innen als bedeutsam angesehen werden. Das Thematisierte ist zumindest zum Teil neu und geeignet, Überzeugungen und Einstellungen $\mathrm{zu}$ verändern, Einsichten und neue Perspektiven zu ermöglichen. Literarische Texte werden also unter anderem deswegen rezipiert, weil das Gelesene aufgrund seines lebensweltlichen Bezuges relevant ist und folglich appliziert werden kann.

Die Relevanz der Gehalte ist nicht losgelöst von den situativen Gegebenheiten. Was ein literarischer Text thematisiert, interessiert nicht oder zumindest nicht in erster Linie, weil derlei die Menschen immer interessiert hat, sondern weil es einen spezifischen Bedarf in einer konkreten historischen Situation gibt. Die Romane aus dem letzten Drittel des 18. Jahrhunderts behandeln nicht Liebe an sich, sie tun dies unter den spezifischen Bedingungen der Zeit. Werthers Liebesideal ist ein Ideal seiner Zeit, ebenso wie das in Millers Siegwart dargestellte oder der Umgang mit der Liebe, welchen Lafontaine vermitteln will. Bei der Rezeption von Reuters Roman ging es nicht um die Frau an sich, bei Mann nicht um das Leben an sich und bei Remarque nicht um den Krieg an sich, sondern immer um spezifische Probleme, die unter spezifischen Umständen diskutiert wurden: die Mädchenfrage, die Mentalität um 1900, der ,Große Krieg‘ mit seinen Folgen für den Einzelnen und die Gesellschaft, von welchen die Zeitgenossen unmittelbar oder mittelbar betroffen waren. Die Rezeption und Applikation ist daher in ihrer konkreten Ausprägung situativ und historisch spezifisch. Dieser situative Rahmen ist die Mikroebene des Alltags, mit ihren Wahrnehmungen, Erfahrungen und Mentalitäten. 
Bei Applikationen kommt des Weiteren ein Faktor zum Tragen, welcher den Umgang mit Literatur betrifft. Literarische Texte sind generisch und konventionell in einem pragmatischen Sinne unterdeterminiert. Es gibt keine allgemeinverbindlichen Regeln dafür, wie Literatur zu lesen ist. Es gibt insbesondere keinen Garanten für die Korrektheit und Geltung des Dargestellten. Als wichtig erweist sich in diesem Zusammenhang die Fiktionalität: Literarische Texte handeln von Fiktivem. Was in ihnen gesagt wird, kann reale Sachverhalte zutreffend wiedergeben, muss es aber nicht. Die Erzähler und Figuren, welche als Träger der Aussagen, der Perspektiven und der wertenden Haltungen fungieren, sind fiktive Instanzen. Das in einem literarischen Text Gesagte ist damit in einem wertneutralen Sinne unverbindlich: Was folgt daraus, wenn eine Figur bestimmte Ansichten äußert, etwa Werther oder Agathe Heidling? Was folgt daraus, wenn der Erzähler eine bestimmte Perspektive entwirft, zum Beispiel Paul Bäumer auf den Krieg oder Michael Berg auf den NS? Was folgt daraus, wenn der Autor mit darstellungstechnischen Mitteln eine bestimmte Aussage nahelegt, etwa Miller und Lafontaine oder Kehlmann? Zunächst einmal nichts. Es bedarf eines Publikums, das die Aussage annimmt oder verwirft, die Perspektive teilt oder ablehnt, sich die Ansicht zu eigen macht oder sie abtut. Neben der Thematik und ihrem situativen Bezug scheint es die relativ große pragmatische Offenheit zu sein, welche die Applikation literarischer Texte befördert.

Eine wichtige Rolle scheint die Eigenaktivität der Rezipient/-innen zu spielen. Der (häufig nicht-bewusste) Akt der Applikation ist das Ergebnis einer eigenen kognitiven Leistung und eines selbstbestimmten Anerkennens oder Ablehnens dessen, was der Text als Option anbietet. Das kann besonders deutlich werden bei der Rezeption von Schlinks Der Vorleser. Nutzer/-innen bei LovelyBooks hoben positiv hervor, dass der Text aufgrund seiner Beschaffenheit, so wie sie sie wahrnahmen, dazu Anlass gibt, sich eine eigene Meinung zu bilden. In die gleiche Richtung geht der häufig vorkommende Hinweis, der Roman rege zum Nachdenken an. Ähnliche Aussagen fanden sich auf der Plattform bei Das Parfum und Die Vermessung der Welt. Wie ein literarischer Text rezipiert und appliziert wird, ist nichts, was den Akteur/-innen widerfährt, sondern verdankt sich einem selbstgefassten Entschluss.

Mitunter wird die Auffassung vertreten, dass die Rezeption literarischer Texte vielgestaltig, ja plural ist. Es gebe, etwas überspitzt gesagt, so viele Rezeptionsmöglichkeiten wie Leser/-innen. Zumindest was die feststellbaren Applikationen der neun untersuchten Romane angeht, erhält man jedoch ein etwas anderes Bild. Es lassen sich durchaus mehrere Applikationen identifizieren, aber in begrenzter Zahl. Selbst bei so vielfältig und kontrovers diskutierten und formal und rhetorisch durchaus komplexen - Romanen wie Aus guter Fami- 
lie und Im Westen nichts Neues gibt es im Wesentlichen drei oder vier distinkte Applikationen, die sich nachweisen lassen. Die Zahl der Applikationen ergibt sich in der Regel daraus, dass unterschiedliche Gehalte eines Textes appliziert werden, nicht daraus, dass der Text radikal anders gelesen würde. Das zeigt sich besonders bei Die Leiden des jungen Werthers und Der Vorleser oder Die Vermessung der Welt. Es kann dabei unterschiedliche Meinungen geben in der Frage, wie ein gegebener Text appliziert werden kann und ob das eine wünschenswerte Applikation ist. Beliebig sind solche Fälle jedoch nicht.

Die festgestellten Applikationen weisen zudem ein in der Regel hohes Maß an Passung mit der Beschaffenheit des Textes auf. Das trifft für die Mehrzahl der Applikationen zu, welche sich bei Goethes Werther, Reuters Aus guter Familie und Remarques Im Westen nichts Neues ausmachen lassen. Manchmal sind die Applikationen, wie gesehen, in ihrer Reichweite beschränkt. Sie beziehen sich dann nur auf Teile oder Details des Textes. Das gilt zum Beispiel für die meisten der Applikationen von Kehlmanns Die Vermessung der Welt. Tatsächlich gibt es Fälle, bei denen die Applikation am Text vorbeigeht. Es leuchtet zum Beispiel nicht ohne Weiteres ein, welche Einsichten in das Wesen des Weiblichen Reuters Roman ermöglicht oder welche Erkenntnisse über das Wesen von Paarbeziehungen oder über Analphabetismus man sich durch die Lektüre von Schlinks Der Vorleser verschaffen kann, wenn man das Gesagte nicht aus seinem Zusammenhang löst. Mitunter werden die Applikationen eher assoziativ anhand

des Textes vorgenommen. Das gilt zum Beispiel für die Allegorisierung von Süskinds Parfum zu einem Roman über den NS. Solche Fälle kommen aber seltener vor.

\subsection{Weiterführende Fragen}

Die zeitgenössische Rezeption der in Kapitel 3 und 4 untersuchten Romane unterscheidet sich vermutlich zumindest in Teilen von der daran anschließenden Rezeptionsgeschichte und zumal von der gegenwärtigen Rezeption. Damit ist eine erste weiterführende Frage benannt: Welche Gemeinsamkeiten und Unterschiede weisen zeitgenössische und rezeptionsgeschichtliche Applikationen auf? Die Art und Weise, wie in den untersuchten Romanen aus dem letzten Drittel des 18. Jahrhunderts über Liebe und Gefühle geschrieben wird, dürfte manchen außerwissenschaftlichen Rezipient/-innen heute fremd anmuten, die Thematik in der dargebotenen Form als weniger relevant erscheinen. Bei Aus guter Familie dürfte überraschen, wie wenig die aus heutiger Sicht in einem weiterreichenden Sinne kritikwürdigen Geschlechterverhältnisse Gegenstand der Rezeption waren. Den Buddenbrooks würden Nutzer/-innen bei LovelyBooks 
und andere vermutlich nicht ohne Weiteres metaphysische Einsichten in das Leben an sich zuschreiben, wie dies um 1900 geschah. Im Westen nichts Neues wird wohl ganz überwiegend als Roman wahrgenommen werden, der zu Recht eine kriegskritische Sicht vermittelt.

Wie wird ein literarischer Text in der Rezeptionsgeschichte und vom heutigen Standpunkt aus appliziert? Dass in diesen Fällen Applikationen vorkommen können, ist in Anbetracht der Ergebnisse dieser Arbeit mit einer hohen Wahrscheinlichkeit anzunehmen. Davon soll anhand von Rezensionen zu Werther, Buddenbrooks und Im Westen nichts Neues, die bei LovelyBooks eingestellt wurden, zumindest ein Eindruck gegeben werden. $\mathrm{Zu}$ den anderen drei Romanen aus dem Korpus gibt es dort keine Rezensionen. Es versteht sich, dass die Beispiele eine eigene Untersuchung der Rezeptionsgeschichte nicht ersetzen können und keine generalisierbaren Aussagen zulassen. Sie dienen lediglich der Illustration und lassen weiterführende Forschungen als aussichtsreich erscheinen.

Im Falle von Goethes Werther verzeichnet LovelyBooks für die Zeit zwischen 2006 und 2020 insgesamt 79 Rezensionen. Fünfzehn davon, also etwas mehr als jede sechste, enthalten Aussagen, die hier einschlägig sind. In einer Rezension wurde der Roman als „Mahnung“ begriffen: „Seht, was passiert, wenn man seinen Gefühlen freien Lauf lässt.“1 Das ist gar nicht so weit von einer der zeitgenössischen Applikationen entfernt. Ein Nutzer meinte, er habe „noch nie so gut erklärt bekommen, warum eine Depression zum Tode führen kann“.'2 Von Einsichten in die Psyche eines Menschen sprachen, obgleich mit anderen Begriffen, bereits die Zeitgenossen. In einer weiteren Rezension wurde das „Thema“ gelobt, „das immer aktuell sein wird: die Liebe“. ${ }^{3}$ Applikation und, allgemein, die Rezeption, muss vom heutigen Standpunkt aus also nicht notwendig anders sein, als die zeitgenössische.

Die Aktualität hoben weitere Nutzer/-innen hervor und betonten dabei gerade den Unterschied zwischen damals und heute. Der Roman sei „sehr aktuell“, weil er im „Kontrast“ stehe „zur heutigen Zeit mit den ganzen Oberfläch-

1 Sakuko: Ein Klassiker. 2016. https://www.lovelybooks.de/autor/Johann-Wolfgang-vonGoethe/Die-Leiden-des-jungen-Werther-142524780-w/rezension/1240501734/ (01.07.2020).

2 Eglfinger: [Rezension von Goethe: Werther]. 2013. https://www.lovelybooks.de/autor/ Johann-Wolfgang-von-Goethe/Die-Leiden-des-jungen-Werther-142524780-w/rezension/ 1020369551/ (01.07.2020).

3 Ein LovelyBooks-Nutzer: [Rezension von Goethe: Werther]. 2012. https://www. lovelybooks.de/autor/Johann-Wolfgang-von-Goethe/Die-Leiden-des-jungen-Werther142524780-w/rezension/950047290/ (01.07.2020). 
lichkeiten“. ${ }^{4}$ Die Darstellung der psychischen Disposition des Protagonisten und seiner Befindlichkeiten sei „genauso, wie es auch heute noch viele Menschen in ihrem Innersten erleben“. ${ }^{5}$ Bisweilen deuten die Texte auf Applikationen hin, die sich eher einem heutigen Standpunkt verdanken und historisch nicht vorkamen. Wenn angenommen wird, dass Werther „die Dinge hinterfragt“, 6 und davon ausgegangen wird, dass der Roman ,gegen festgesetzte Normen und Konventionen“ gerichtet ist, ${ }^{7}$ dann wird dem Text eine kritische Perspektive zugeschrieben, die zeitgenössisch nicht eingenommen wurde. In einer Rezension wurde lobend hervorgehoben: „Hier dürfen Männer noch Gefühle zeigen“. ${ }^{8}$ Damit stellte die Nutzerin den Text in einen Zusammenhang, der zeitgenössisch so nicht gegeben war. Daneben gibt es solche, die dem Roman jegliche Relevanz für die Gegenwart absprechen: „Man sollte neuere und aktuelle Themen durchgehen in Schulen und nicht so etwas. “9

$\mathrm{Zu}$ den Buddenbrooks liegen für den Zeitraum von 2006 bis 2019 insgesamt 107 Rezensionen vor, von denen allerdings nur etwa jede dreizehnte, nämlich acht, einschlägige Aussagen enthält. Die geringe Zahl kann als Indiz gewertet werden, dass der Roman heute kein großes Applikationspotenzial hat, zumindest bei diesen Rezipient/-innen. Eine Nutzerin schrieb am Ende ihrer Rezension, sie sei „froh [...], nicht als Frau im 19. Jahrhundert leben zu müssen“. ${ }^{10}$ Das ist zwar keine besonders komplexe Aussage, aber doch eine, die darauf hinweist, dass die Nutzerin den Roman im Sinne des in Kapitel 4.2.3 identifizierten

4 Stephanus: Ein immer noch aktuelles Meisterwerk. 2013. https://www.lovelybooks.de/ autor/Johann-Wolfgang-von-Goethe/Die-Leiden-Des-Jungen-Werthers-142524780-w/rezension/ 1046427348/ (01.07.2020).

5 Raven: [Rezension von Goethe: Werther]. 2007. https://www.lovelybooks.de/autor/JohannWolfgang-von-Goethe/Die-Leiden-des-jungen-Werther-142524780-w/rezension/986296233/ (01.07.2020).

6 Chiaramaus: Ein sehr gelungener Briefroman! 2016. https://www.lovelybooks.de/ autor/Johann-Wolfgang-von-Goethe/Die-Leiden-des-jungen-Werther-142524780-w/rezension/ 1359966935/ (01.07.2020).

7 Ambermoon: Liebe, Schmerz und Melancholie in seiner ausdrucksstärksten Form. 2016. https://www.lovelybooks.de/autor/Johann-Wolfgang-von-Goethe/Die-Leiden-des-jungenWerther-142524780-w/rezension/1238724224/ (01.07.2020).

8 Fiona89: [Rezension von Goethe: Werther]. 2010. https://www.lovelybooks.de/autor/ Johann-Wolfgang-von-Goethe/Die-Leiden-des-jungen-Werther-142524780-w/rezension/ 967240707/ (01.07.2020).

9 Aniela: [Rezension von Goethe: Werther]. 2017. https://www.lovelybooks.de/autor/JohannWolfgang-von-Goethe/Die-Leiden-des-jungen-Werther-142524780-w/rezension/1447110155/ (01.07.2020).

10 Farbewirbel: Zähne, Bärte, Wetterlage. 2017. https://www.lovelybooks.de/autor/ThomasMann/Buddenbrooks-63523196-w/rezension/1445330437/ (01.07.2020). 
Applikationspotenzials gelesen hat: Tony, auf die sie die Aussage wohl bezieht, scheitert ja tatsächlich, wie zu zeigen versucht, an den geschlechts- und milieuspezifischen Rollenerwartungen, denen sie gerecht zu werden versucht, und die man aus heutiger Sicht wohl mehrheitlich ablehnen würde. Der Roman verfügt in dieser Hinsicht noch und vielleicht gerade heute über ein Applikationspotenzial. Es lassen sich Beispiele finden, die eine gesellschaftskritische Lesart und damit verbundene Applikationen nahelegen, die sich allerdings weniger der Beschaffenheit des Textes verdankt, sondern eher der heutigen Situation. Eine Nutzerin meinte, der Roman habe „nichts von seiner Aktualität eingebüßt, besonders wenn ich an Börsenspekulanten und all die Konsumenten der Unterhaltungs- und Trösterchenindustrie denke“. ${ }^{11}$ Solche Bezüge können wohl nur vom heutigen Standpunkt aus hergestellt werden. Eine Aussage, wonach „Thomas Mann unter anderem die Dekadenz der damaligen Oberschicht an den Pranger stellt“, ist jedenfalls historisch wohl nicht korrekt. ${ }^{12}$ In einer anderen Rezension war von „einer starken Gesellschaftskritik“ des Romans die Rede. ${ }^{13}$ Das Gelesene so zu applizieren, setzt es in einer Art und Weise mit einer heutigen Sicht auf die damaligen Verhältnisse in Beziehung, die durchaus möglich ist, aber in dieser Form keiner historisch nachweisbaren Rezeption und Applikation entspricht.

$\mathrm{Zu}$ Im Westen nichts Neues gibt es bei LovelyBooks insgesamt 83 Rezensionen, von denen 46, also etwas mehr als die Hälfte, deutliche Applikationen aufweisen. Sie stammen ebenfalls aus dem Zeitraum von 2006 bis 2020. Es gibt einen weitreichenden Konsens in der Wahrnehmung des Buches und in der Applikation des Gelesenen, wie zumindest kurz zu umreißen ist. Der Roman wird als zutreffende Schilderung des tatsächlichen Kriegsgeschehens und des Leidens der Soldaten angesehen, er zeige dessen Brutalität und Sinnlosigkeit, vermittle eine antiheroische und pazifistische Sicht, sei erschütternd und bewegend, könne Überzeugungen und Einstellungen ändern, diene der Erinnerung und Mahnung. Ausgewählte Beispiele sollen verdeutlichen, wie die Applikationen konkret aussehen.

11 KristinaTiemann: Buddenbrooks sind aktuelle Zeitgeschichte. 2017. https://www. lovelybooks.de/autor/Thomas-Mann/Buddenbrooks-63523196-w/rezension/1413404723/ (01.07.2020).

12 Ein LovelyBooks-Nutzer: Literaturwelt, verzeih mir. 2014. https://www.lovelybooks.de/ autor/Thomas-Mann/Buddenbrooks-63523196-w/rezension/1089776456/ (01.07.2020).

13 Samtpfote: [Rezension von Mann: Buddenbrooks]. 2012. https://www.lovelybooks.de/ autor/Thomas-Mann/Buddenbrooks-63523196-w/rezension/1008699613/ (01.07.2020). 
In einer Rezension hieß es: „Wer noch nicht überzeugt davon ist, wie sinnlos Kriege sind, der sollte dieses Buch lesen." ${ }^{14}$ Es wurde also davon ausgegangen, dass der Roman die Überzeugungen in dieser Hinsicht verändern kann. In einer anderen Rezension wurde festgestellt: „Man kann es sicherlich als Antikriegsbuch bezeichnen, aber nicht, weil die Protagonisten darin pazifistische Parolen von sich geben, sondern weil es einem einfach zeigt, wie Krieg ist: grausam und verstörend. "15 Dem Roman wurde also aufgrund der Art und Weise, wie der Krieg geschildert wird, das Potenzial zugeschrieben, zu der Einsicht zu führen, wie der Krieg wirklich ist. Eine Nutzerin schrieb: „[D]urch diesen Roman bekenne ich mich jetzt vollständig als Pazifistin“. ${ }^{16}$ Eine andere meinte: „Danach muss man einfach zum Pazifisten werden!“17 $̈$ Ähnlich eine dritte Rezension: „,Nie wieder Krieg!‘ sind sicherlich die Gedanken eines jeden, der dieses Buch gelesen hat. “18 In diesen Rezensionen wird mit Blick auf die eigene Person oder allgemein festgestellt, dass die Lektüre des Romans zu einer pazifistischen Haltung führen kann, wenn nicht muss. An einer Stelle wurde gesagt: „Der Erste Weltkrieg - und Kriege im Allgemeinen - werden oft romantisiert. Remarque öffnet mit seinem Werk Im Westen nichts Neues dem Zuhörer die Augen und überzeugt ihn vom Gegenteil.“" ${ }^{19}$ Der Roman ist hier also geeignet, ein korrektes Bild davon zu vermitteln, was Krieg sei, und jeglicher Idealisierung $\mathrm{zu}$ wehren. Eine Nutzerin betrachtete das Buch als „Mahnung“ und als „Erinnerung an alle, die wie ich in Frieden aufwachsen durften, diesen zu schätzen und zu bewahren“. ${ }^{20}$ Aus der eigenen Sicht geschrieben, aber mit wei-

14 Itwt69: Die verlorene Generation. 2016. https://www.lovelybooks.de/autor/Erich-MariaRemarque/Im-Westen-nichts-Neues-48333411-w/rezension/1407631077/ (01.07.2020).

15 Waffelkuchen: [Rezension von Remarque: Im Westen nichts Neues]. 2011. https://www. lovelybooks.de/autor/Erich-Maria-Remarque/Im-Westen-nichts-Neues-48333411-w/rezension/ 966802784/ (01.07.2020).

16 Gilfaen: [Rezension von Remarque: Im Westen nichts Neues]. 2011. https://www. lovelybooks.de/autor/Erich-Maria-Remarque/Im-Westen-nichts-Neues-48333411-w/rezension/ 966820110/ (01.07.2020).

17 Eva1982: [Rezension von Remarque: Im Westen nichts Neues]. 2008. https://www. lovelybooks.de/autor/Erich-Maria-Remarque/Im-Westen-nichts-Neues-48333411-w/rezension/ 985758104/ (01.07.2020).

18 Jens65: [Rezension von Remarque: Im Westen nichts Neues]. 2008. https://www. lovelybooks.de/autor/Erich-Maria-Remarque/Im-Westen-nichts-Neues-48333411-w/rezension/ 985701686/ (01.07.2020).

19 Julia7717: Die Schrecken des Ersten Weltkrieges. 2018. https://www. lovelybooks.de/autor/E.M.-Remarque/Im-Westen-nichts-Neues-1075757887-w/ (01.07.2020). 20 Anna_Ressler: [Rezension von Remarque: Im Westen nichts Neues]. 2018. https://www. lovelybooks.de/autor/E.M.-Remarque/Im-Westen-nichts-Neues-1075757887-w/ (01.07.2020). 
tereichendem Anspruch, nahm sie an, dass der Roman zu der Einsicht führe, wonach Frieden schätzenswert und bewahrenswert ist. Besonders deutlich wird die Applikation in einer Rezension, die explizit den Bezug zur Gegenwart herstellt. Die Nutzerin gibt zu bedenken, „dass es immer noch überall auf der Welt Menschen gibt, die Ähnliches oder gar Schlimmeres erleiden“. ${ }^{21}$ Es liegt auf der Hand, dass mit den zitierten Beispielen eine der zeitgenössisch möglichen Applikationen realisiert wird. War die Meinung Ende der 1920er Jahre jedoch gespalten und polarisiert, so gibt es in der gegenwärtigen Wahrnehmung, wie sie sich in den Rezeptionsdokumenten darstellt, nur eine.

Es zeigt sich, was unabhängig von diesen der Illustration dienenden Beispielen vermutet werden konnte. In der Rezeptionsgeschichte lassen sich Applikationen ausmachen. Tentativ kann man vier Fälle unterscheiden. Erstens: Eine Applikation, welche zur Zeit der Erstrezeption vorkam, kann auch in der Rezeptionsgeschichte realisiert werden. Das betrifft zum Beispiel Einsichten in psychische Befindlichkeiten und den Umgang mit Gefühlen (Goethes Werther) oder die Übernahme einer kriegskritischen oder pazifistischen Einstellung (Remarques Im Westen nichts Neues). Dabei sollten die Unterschiede nicht aus dem Blick geraten. Werthers Melancholie ist etwas Anderes als Depression. Zeitgenössische Kritik daran, wie der Erste Weltkrieg geführt wurde, ist nicht mit einer prinzipiell kriegskritischen oder gar pazifistischen Sicht zu verwechseln. Andere Applikationen werden, zweitens, unter gewandelten Umständen nicht mehr vorgenommen. Es wäre wohl überraschend, wenn das heutige Publikum die Buddenbrooks im Geiste einer um 1900 herrschenden Mentalität betrachtete. Drittens: Aus den Zusammenhängen seiner Erstrezeption gelöst, kann ein literarischer Text Anlass sein zu Applikationen, die zeitgenössisch nicht vorkamen, aber möglich waren. Das trifft zum Beispiel für die Annahme zu, wonach Manns Roman das Problem geschlechtsspezifischer Rollenerwartungen deutlich mache. Viertens können zu einem späteren Zeitpunkt Applikationen vorkommen, die zur Zeit der Erstrezeption nicht möglich waren. Das gilt offensichtlich zum Beispiel da, wo der historisch fremde Text im Kontrast steht zur heutigen Situation, oder da, wo einem vom heutigen Standpunkt aus etwas am Text als kritikwürdig erscheint, für das die Zeitgenossen kein Bewusstsein hatten.

$\mathrm{Zu}$ den weiterführenden Fragen gehört des Weiteren die nach generischen und epochalen Spezifika. Eine größer angelegte Studie könnte eventuell zeigen, dass es einen gewissen Zusammenhang gibt zwischen Applikationen und bestimmten Gattungen oder Epochen. Allgemeine Verlaufsvorstellungen über den

21 Rabentocher: Mir fehlen die Worte. 2016. https://www.lovelybooks.de/autor/E.M.Remarque/Im-Westen-nichts-Neues-1075757887-w/ (01.07.2020). 
Gang der Literaturgeschichte und Einsichten in die Wichtigkeit generischer Zugehörigkeit machen einen solchen Zusammenhang wahrscheinlich. Es steht $\mathrm{zu}$ vermuten, dass sich manche Gattungen auf unterschiedliche Weise und für jeweils andere Applikationen eignen. Zu erinnern ist aber an den Umstand, dass die prinzipielle Eignung literarischer Texte für Applikationen aus generischen Eigenschaften hervorgeht, die für literarische Texte allgemein charakteristisch sind. Ebenso dürften sich zwischen den Epochen Unterschiede ergeben. Man könnte zum Beispiel annehmen, dass im 18. Jahrhundert ethische Applikationen wichtiger waren als $\mathrm{zu}$ anderen Zeiten und eine gegenüber moderner Literatur weniger komplexe Form annahmen. Für solche literaturgeschichtlichen Aussagen erweist sich das Korpus dieser Arbeit als nicht umfangreich genug. Es erlaubt allerdings die Feststellung einer zentralen Gemeinsamkeit: Applikationen kommen bei allen Romanen vor und sie weisen manche Ähnlichkeiten auf. Applikation als Praktik des Umgangs mit Literatur hat eine lange Dauer. Als historische Praktik ist sie nicht zeitenthoben, dafür aber relativ zeitbeständig. Ein barocker Roman funktioniert offensichtlich fundamental anders als ein postmoderner. Etwaige Applikationen dürften in ihren speziellen Ausprägungen verschieden sein. Aber dass beide so unterschiedlichen Texte von dem jeweiligen zeitgenössischen Publikum appliziert werden können, davon ist aufgrund der Ergebnisse dieser Arbeit auszugehen.

Ferner wäre eine eingehendere historische Einbettung der Applikation wünschenswert, indem sie mit anderen Praktiken und auf den Umgang mit Literatur einwirkenden Faktoren in Beziehung gesetzt wird. Applikation ist zudem nicht auf Literatur beschränkt, andere Medien wie zum Beispiel Filme können ebenfalls zu Applikationen Anlass geben. Tatsächlich dürfte das für jede Kunstform gelten, die Gehalte vermittelt. Aufschlussreich könnte es nicht zuletzt sein, Applikationen in einem bestimmten institutionellen Rahmen der Literaturvermittlung, etwa der Schule, zu untersuchen.

Für weiterführende Fragen dieser Art sollte diese Studien Voraussetzungen schaffen. Literatur, so zeigt es die vorliegende Arbeit, ist dazu da, verwendet, gebraucht und angewandt, also appliziert zu werden. Jedenfalls ist es das, was zahlreiche Rezipient/-innen in Geschichte und Gegenwart beim Umgang mit Literatur machen. Grund genug, die Applikation ernst zu nehmen und literaturwissenschaftlich zu untersuchen, wie es hier geschehen ist. 
